\title{
A correlation analysis method for analyzing tribological states using acoustic emission, frictional coefficient, and contact resistance signals
}

\author{
Pengyi TIAN, Yu TIAN*, Lei SHAN, Yonggang MENG, Xiangjun ZHANG \\ State Key Laboratory of Tribology, Tsinghua University, Beijing 100084, China \\ Received: 24 April 2014 / Revised: 30 June 2014 / Accepted: 27 September 2014 \\ (C) The author(s) 2014. This article is published with open access at Springerlink.com
}

\begin{abstract}
A multi-physical signal correlation analysis method is proposed to identify the different tribological properties of materials. The acoustic emission (AE), contact resistance, and frictional force behaviors during dry sliding between four metals, 45\# carbon steel, YG12 carbide, 2A12 aluminum alloy, and H62 brass, have been studied. Both positive and negative correlations between the root mean square of the amplified AE (AE RMS) signal and the frictional coefficient have been found in the experiments. In addition, the AE RMS signal and the contact resistance changed with changing sliding speed and normal load in different ways. The different correlation behaviors have been attributed to diverse tribological states under different experimental conditions due to different material characteristics. The correlation analysis provides a new method of quantitatively identifying the tribological states and the AE sources during frictional interaction. The observed anomalous correlations between the AE signal and frictional coefficient should be properly considered according to the different material properties during industrial friction condition monitoring using AE technology.
\end{abstract}

Keywords: acoustic emission; correlation analysis; dry friction; contact resistance

\section{Introduction}

Because the interfacial friction process is quite complex and the states of the mating surfaces cannot be easily observed directly, researchers have been committed to developing various experimental methods and equipment to study the nature of this process. Various tribotesters have been used to measure the frictional coefficient on a macroscopic scale [1]. Atomic-force microscopy [2-5] and friction-force microscopy [6, 7] have made it possible to study friction, wear, and lubrication on an atomic scale. Quartz crystal microbalance [8-10] was developed to study the tribological characteristics of a single-layer molecular film under boundary lubrication. Several microscopic techniques, such as scanning electron microscopy (SEM) [11] and white light interferometry [12], have been used for

\footnotetext{
* Corresponding author: Yu TIAN.

E-mail: tianyu@mail.tsinghua.edu.cn
}

surface morphology measurements after friction testing. However, the above mentioned conventional methods are only able to measure the mechanical force during friction testing or conduct offline measurements after testing. Because various complex physical-chemical processes simultaneously occur during interfacial friction [13], comprehensive detection of these physical phenomena is essential for evaluating real-time states during the frictional interaction of surfaces. Numerous experimental studies have been conducted for each of these phenomena, including frictional heat [14, 15], frictional noise [16], triboelectrification [17], triboluminescence [18], etc. However, most of the studies were concerned with only a single phenomenon, and a combination of physical phenomena has been rarely considered.

Tribological research has evolved from qualitative to quantitative and from single-field to multi-field [19]. A comprehensive study of several physical phenomena 
that occur during dry sliding was conducted in the present work. In addition to conventional measurements of the frictional force and coefficient, a nondestructive testing method denoted as acoustic emission (AE) detection was also used. AE is a phenomenon resulting from the production of transient elastic waves at the local contact regions of materials due to rapid elastic/plastic energy release [20]. This phenomenon widely occurs during interfacial friction because of the contacts and deformations of local asperities of the rough surfaces. The characteristics of AE during frictional interaction and wear have been extensively investigated for better utilization of $\mathrm{AE}$ technology in industrial applications [21, 22]. Hase et al. [23-26] examined the relationships between $\mathrm{AE}$ signals and different wear states such as adhesive and abrasive wear, and mild and severe wear. Furthermore, theoretical models have been established in an effort to calculate the AE power expended during frictional interaction [27, 28]. Another physical signal measured in the present study was the contact electrical resistance, which is closely related to the contacting state. Because the actual contact area is a small percentage of the apparent area, the number, size, and distance of separation of the individual contact areas and the electrical properties of the metal involved will all affect the contact resistance [29]. A considerable amount of theoretical research has been conducted on the relationship between the actual contact area and the contact resistance [30-32].

However, previous research has been mostly concerned with only the AE signal or only the contact resistance. To link various physical phenomena to the microscopic mechanism of friction, a correlation analysis method is proposed in this paper to quantitatively determine the different tribological states during dry sliding. The correlation analysis among $\mathrm{AE}$, contact resistance, and the frictional coefficient indicate that different correlations correspond to different tribological behaviors of the materials.

\section{Experimental apparatus and procedures}

\subsection{Experimental apparatus}

Figure 1 shows the experimental system. The main component was the universal micro-tribotester (UMT-2;

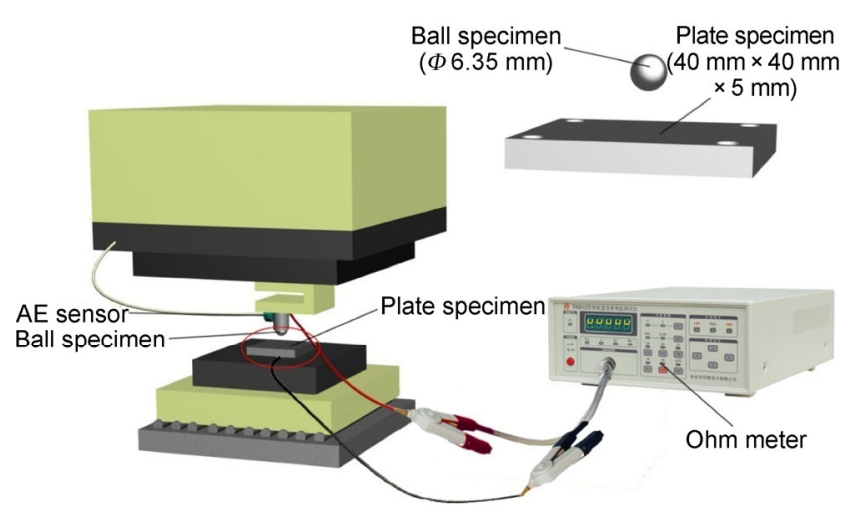

Fig. 1 Schematic of the experimental system.

Center for Tribology, Inc.). Reciprocating sliding motion was achieved by a crank-slider mechanism. The plate specimen was fixed to the precision linear stage, which was connected to a rod driven by a motor. The reciprocating stroke was adjustable by changing the length of the crank. The upper specimen was clamped in a ball holder attached to a stationary suspension mount. The suspension mount was connected to a two-dimensional load sensor.

A wideband AE sensor (CETR AE-5; Center for Tribology, Inc.) was used to measure the acoustic emission signals during friction testing. The AE sensor was integrated with the UMT-2 tribotester. The working surface of the sensor employed is composed of a piezoelectric ceramic that converts stress waves into voltage signals. The operational frequency range of this sensor is from $0.2 \mathrm{MHz}$ to $5 \mathrm{MHz}$, and the resonant frequency is $400 \mathrm{kHz}$. The sensor was coupled onto the side of the ball holder with petroleum jelly. A signal amplifier was mounted inside the signal conditioning box on the UMT-2 carriage, and the sensor cable connected to the amplifier's input. The AE signals were amplified and filtered by a plug-in module that could accommodate various frequencies. The root mean square of the amplified AE signal (AE RMS) was converted to DC and connected to the data acquisition system for display, storage, and analysis. The AE amplifier gain was $60 \mathrm{~dB}$, and the RMS output time was $0.5 \mathrm{~ms}$. Contact resistance was measured using the Kelvin four-wire method with a auto DC-LowOhmmeter (TH2512; Changzhou Tonghui Electronic Co., Ltd.). This system has a resolution of $1 \mu \Omega$ and nine measuring ranges from $20 \mathrm{~m} \Omega$ to $2 \mathrm{M} \Omega$. The sampling frequency can be chosen as $2.5 \mathrm{~Hz}$ or $10 \mathrm{~Hz}$. 


\subsection{Test specimens}

With consideration for various industrial processes such as cutting and grinding and commonly used mechanical components that are closely related to tribology, four metals were used in our tribological tests, namely, 2A12 aluminum alloy, H62 brass, 45\# carbon steel, and YG12 carbide. Table 1 lists the physical properties of these alloys. The ball samples had a diameter of $6.35 \mathrm{~mm}$, and the plate samples had a geometric specification of $40 \mathrm{~mm} \times 40 \mathrm{~mm} \times$ $5 \mathrm{~mm}$. The surface roughness of the specimens was about $0.25 \mu \mathrm{m}$.

\subsection{Experimental procedure}

The specimens were ultrasonically cleaned with acetone followed by ethanol for $10 \mathrm{~min}$ prior to each test. The reciprocating distance was set to $8 \mathrm{~mm}$. The

Table 1 Properties of the specimen materials.

\begin{tabular}{ccccc}
\hline & $\begin{array}{c}\text { 2A12 } \\
\text { aluminium } \\
\text { alloy }\end{array}$ & $\begin{array}{c}\text { H62 } \\
\text { brass }\end{array}$ & $\begin{array}{c}45 \# \\
\text { carbon teel }\end{array}$ & $\begin{array}{c}\text { YG12 } \\
\text { carbide }\end{array}$ \\
\hline $\begin{array}{c}\text { Hardness } \\
\text { HV137 }\end{array}$ & HV172 & HV600 & HV1300 \\
$\begin{array}{c}\text { Young's modulus } \\
(\mathrm{GPa})\end{array}$ & 70 & 100 & 209 & 470 \\
$\begin{array}{c}\text { Poisson's ratio } \\
\text { Electric resistivity } \\
(\mathrm{n} \Omega \cdot \mathrm{m})\end{array}$ & 0.31 & 0.364 & 0.274 & 0.23 \\
$\begin{array}{c}\text { Melting point }\left({ }^{\circ} \mathrm{C}\right) \\
\text { Thermal }\end{array}$ & 638 & 71 & 320 & 206 \\
$\begin{array}{c}\text { Thenductivity } \\
(\mathrm{W} / \mathrm{mK})\end{array}$ & 193 & 108.86 & 48 & 72 \\
$\begin{array}{c}\text { Specific heat } \\
\text { capacity (J/kgK) }\end{array}$ & 924 & 393.6 & 472 & $\sim 200-$ \\
\hline
\end{tabular}

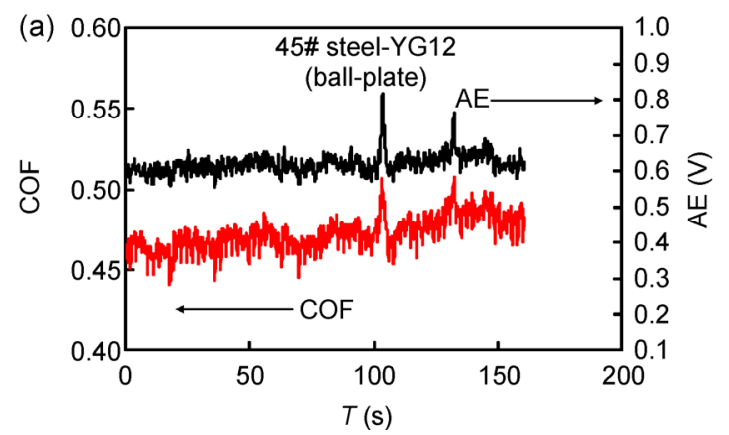

samples were preconditioned by sliding at a frequency of $5 \mathrm{~Hz}(1.33 \mathrm{~mm} / \mathrm{s}$ ) for $5 \mathrm{~min}$ under a load of $2 \mathrm{~N}$ (for 2A12 and H62) or $5 \mathrm{~N}$ (for YG12 and 45\# steel) prior to each test. The load was set to $2,5,7$, and $10 \mathrm{~N}$ and the sliding speed was set to $16,40,64,88,112,136$, and $160 \mathrm{~mm} / \mathrm{s}$ for each load. The tribotester ran for $180 \mathrm{~s}$ at each speed and then accelerated to the next speed over a period of $5 \mathrm{~s}$. The ohmmeter sampling rate was $10 \mathrm{~Hz}$. All experiments were conducted under dry sliding conditions at room temperature (about $25^{\circ} \mathrm{C}$ ) and a relative humidity of about $20 \%$.

\section{Experimental results and discussions}

\subsection{Correlation analysis}

$\mathrm{AE}$ events during frictional interaction are caused by the deformations and fractures of countless asperities, and are, therefore, continuous signals. The AE RMS is then used for analysis. Variations of the frictional coefficient and AE RMS with time were found to differ according to the tribological states. Generally, two correlation types between the AE RMS and the frictional coefficient were observed in our experiments. Typical experimental results for these two types of correlation are shown in Fig. 2. For the carbide ballcarbide plate pair (in the following, the frictional pairs are all expressed in the order ball-plate), a positive correlation existed between the two signals, as shown in Fig. 2(a). The AE RMS signal almost exactly traced the frictional coefficient. However, for the brassaluminum alloy pair, the AE RMS and the frictional coefficient presented a negative correlation with larger fluctuations, as shown in Fig. 2(b).

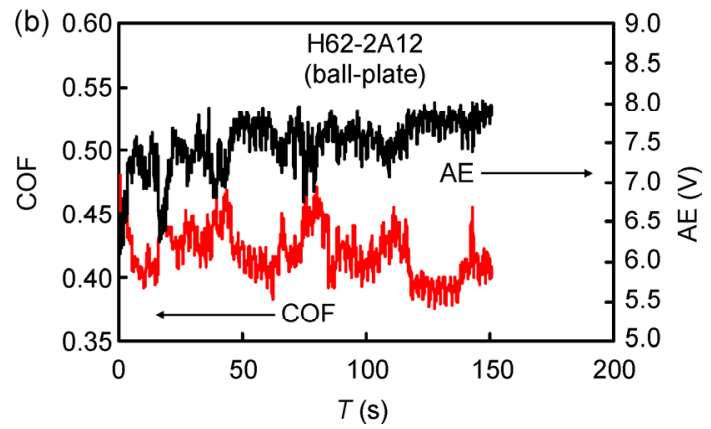

Fig. 2 Friction coefficient and AE RMS signal vs. time during dry friction. (a) 45\# steed- YG12, speed = $112 \mathrm{~m} / \mathrm{s}$, Fn = $5 \mathrm{~N}$; (b) H62$2 \mathrm{~A} 12$, speed $=112 \mathrm{~m} / \mathrm{s}, \mathrm{Fn}=5 \mathrm{~N}$. 
To quantitatively evaluate the different relationships between the two physical signals, correlation analysis for the frictional pairs under equivalent load and speed conditions was conducted using the following equation:

$$
r=\frac{\sum_{i=1}^{n}\left(x_{i}-\bar{x}\right)\left(y_{i}-\bar{y}\right)}{\sqrt{\sum_{i=1}^{n}\left(x_{i}-\bar{x}\right)^{2} \sum_{i=1}^{n}\left(y_{i}-\bar{y}\right)^{2}}}
$$

where $r$ is the correlation coefficient, $x_{i}$ and $\bar{x}$ are the value of each data point and the mean value of one of the signals, respectively (either the frictional coefficient [COF] or contact resistance [RES]), and $y_{i}$ and $\bar{y}$ are the value of each data point and the mean value of the AE RMS signal.

The correlation coefficient of the data shown in Fig. 2(a) was 0.79 , indicative of a highly positive correlation between the two signals. For the frictional pair in Fig. 2(b), the correlation coefficient was -0.85 . The correlation coefficients for the four metal pairs under different sliding speeds and loads were calculated and are presented in Fig. 3. For different metal frictional pairs, the correlation curves can be generally divided into two types. For the metal pairs shown in Figs. 3(a) and 3(b), which were defined as Type I, the correlation coefficients $r_{\mathrm{AE}-\mathrm{COF}}$ were negative over a wide speed range. At low speeds, the AE RMS was more negatively correlated with the frictional coefficient as the speed increased. At high speeds, $r_{\mathrm{AE}-\mathrm{COF}}$ slightly increased with increasing sliding velocity. Whereas, for some other metal pairs such as carbide and $45 \#$ steel, as shown in Figs.3(c) and 3(d), which were defined as Type II, $r_{\mathrm{AE}-\mathrm{COF}}$ were mostly positive and increased with increasing sliding velocity until reaching a relatively stable value close to 1 . For all the metal pairs tested, $r_{\mathrm{AE}-\mathrm{COF}}$ mainly depended on the sliding velocity, and was nearly unaffected by variations in the normal load.

To further analyze the tribological states, correlation analysis was also conducted between the AE RMS and the contact resistance, which is closely related to the real contact area and state. The correlation coefficients $r_{\mathrm{AE}-\mathrm{RES}}$ are shown in Fig. 4 . For the Type I metal pairs, despite the large fluctuations, an opposite trend to that of $r_{\mathrm{AE}-\mathrm{COF}}$ can be observed. At low speeds, $r_{\mathrm{AE}-\mathrm{RES}}$ increased with increasing velocity, but began to decrease at high speeds. However, the $r_{\mathrm{AE}-\mathrm{RES}}$ curves for the Type II metal pairs exhibited a different trend. At the lowest speed $(16 \mathrm{~mm} / \mathrm{s})$, the AE signals were so weak that the calculated correlation coefficient $r_{\mathrm{AE}-\mathrm{RES}}$ was absolutely low, and, at relatively higher speeds (albeit, still low), $r_{\mathrm{AE}-\mathrm{RES}}$ was positive. With
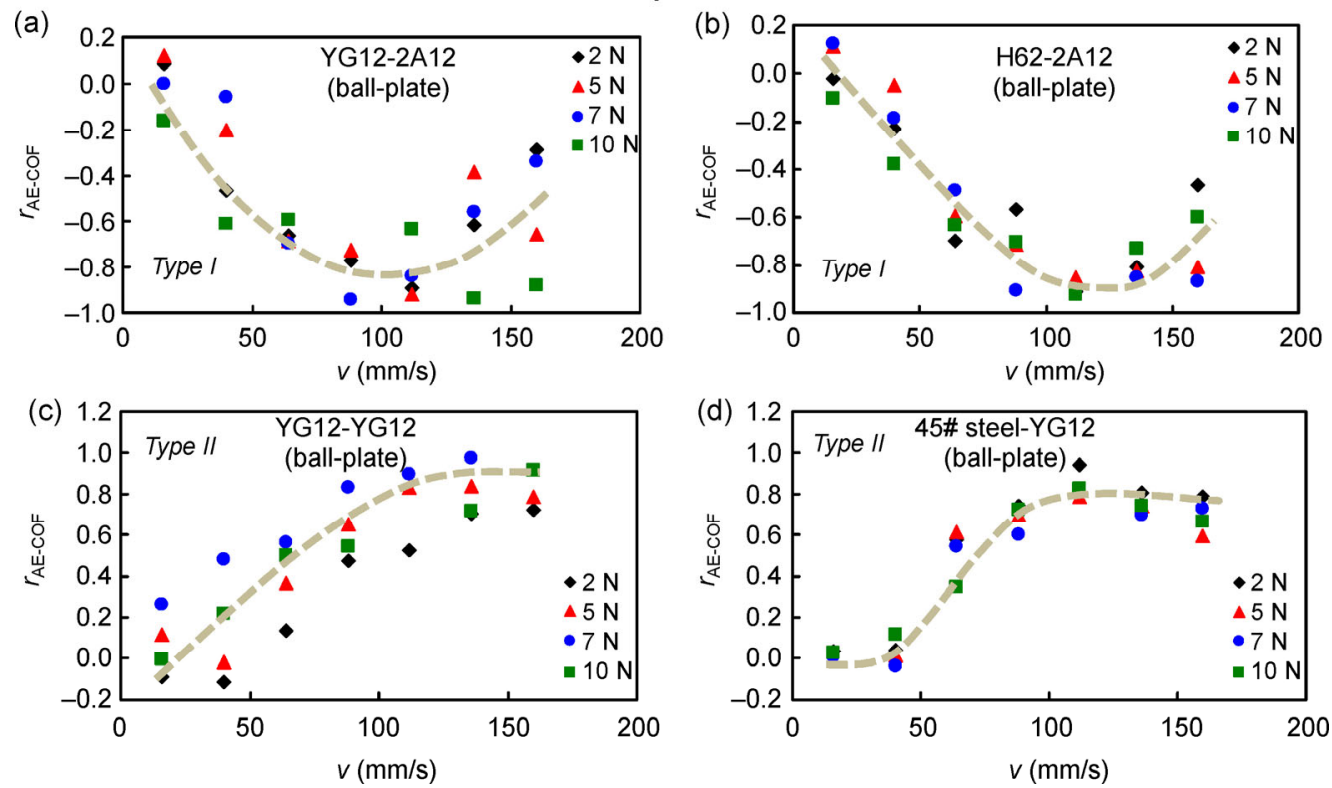

Fig. 3 Correlation coefficients of the AE signal and friction coefficient at different speeds. (a)-(d): YG12-2A12, H62-2A12, YG12YG12, 45\# steel-YG12. 

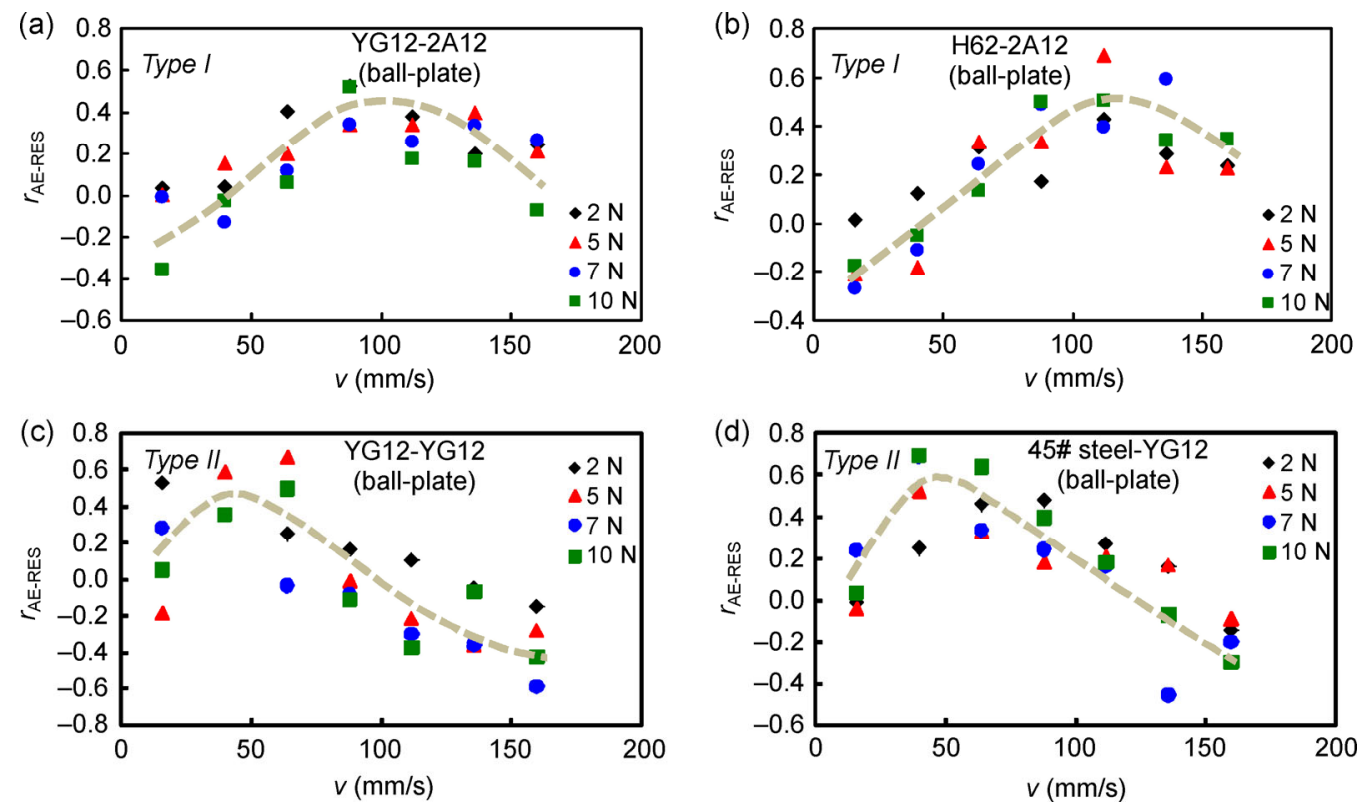

Fig. 4 Correlation coefficients of AE and contact resistance at different speeds. (a)-(d): YG12-2A12, H62-2A12, YG12-YG12, 45\# steel-YG12.

increasing speed, $r_{\mathrm{AE}-\mathrm{RES}}$ decreased and the contact resistance and AE RMS became more and more negatively correlated.

\subsection{Variations of AE RMS and the contact resistance with sliding speed and load}

For the metal pairs of the two correlation types, the AE RMS and the contact resistance were also found to change with speed and load in different ways. The mean values of the AE RMS of these metal pairs under different frictional conditions are shown in Fig. 5. The AE RMS signals significantly increased with increasing sliding speed for all of the frictional pairs tested. However, for the Type I metal pairs, YG12-2A12 and H62-2A12 (Figs. 5(a) and 5(b)), the AE RMS was nearly independent of the applied load. Whereas, for the other two pairs (Figs. 5(c) and 5(d)) whose correlation curves were Type II, the AE RMS signals significantly increased with increasing normal load.

Figure 6 shows the electrical resistance curves with increasing sliding velocity under different loads for the four metal pairs tested. For the Type I metal pairs, YG12-2A12 and H62-2A12, the contact resistances remained at low values except at high speed under a very low load. This result is indicative of only a small change in the real contact area with increasing normal load. Meanwhile, for the Type II metal pairs, YG12YG12 and 45\# steel-YG12, the contact resistances were significantly reduced with increasing normal load. This indicates that the actual contact areas of these frictional pairs significantly increased with increasing normal load.

\subsection{Discussion}

Experiments were conducted with frictional pairs that exchanged the materials of the ball and the plate (e.g., 2A12-H62 and YG12-45\# steel), and similar results were obtained. Therefore, the different correlation types and tribological behaviors are considered to be related to the various wear states and AE sources due to the different material structures and physical properties.

To observe the different wear states, the worn surfaces of these specimens were examined by SEM. Typical worn surface morphologies for the two pair types are shown in Fig. 7. Significant plastic flow of the surface material can be observed for the Type I metal (brass and aluminum alloy) surfaces. In contrast, the worn surfaces of the Type II materials (carbide and 45\# steel) mainly exhibited elastic/plastic compression and partial peeling. In addition, the micrographs of the wear particles provide further indication of two 

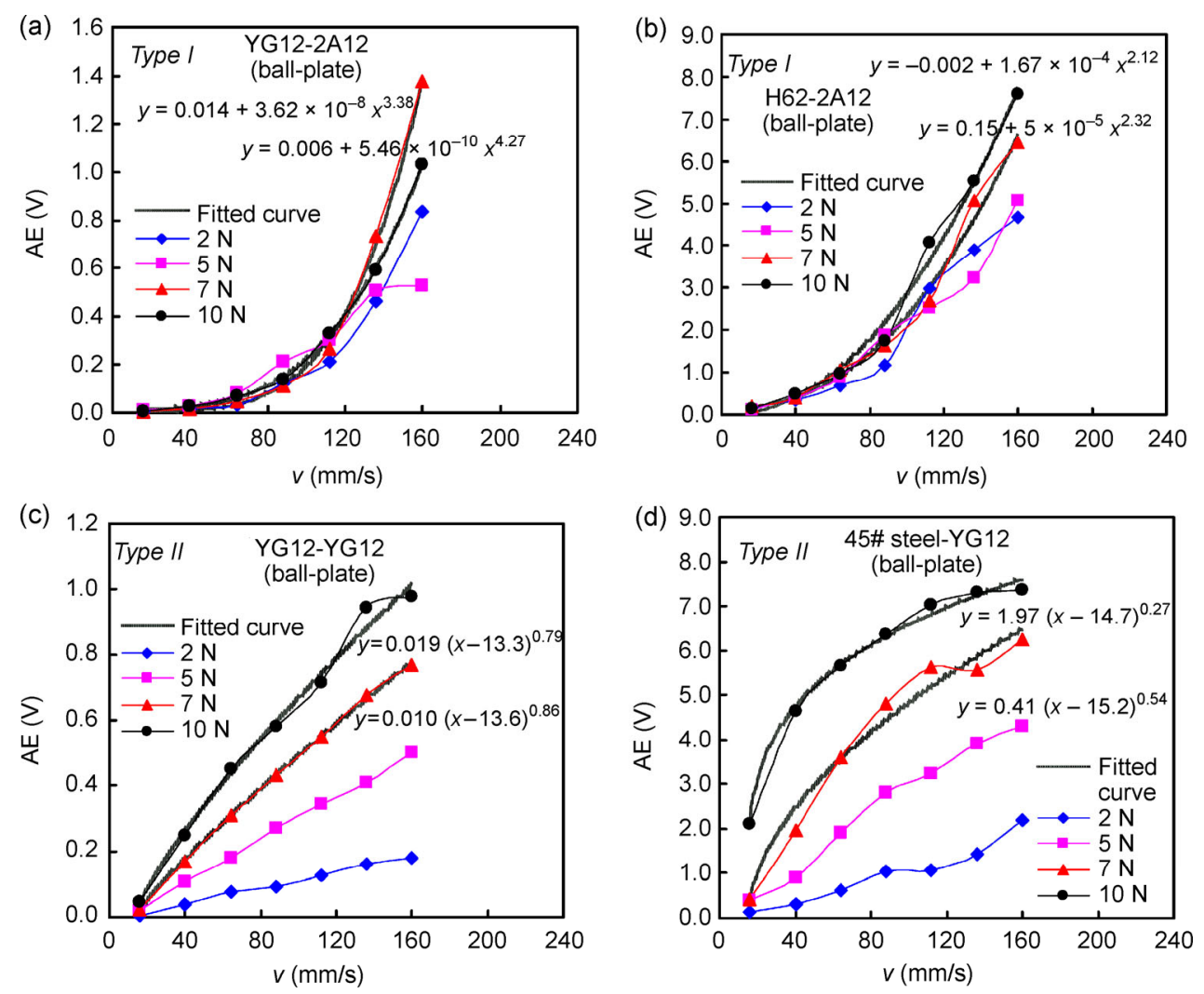

Fig. 5 Mean AE RMS signals vs. speed in dry friction under different loads. (a)-(d): YG12-2A12, H62-2A12, YG12-YG12, 45\# steel-YG12.
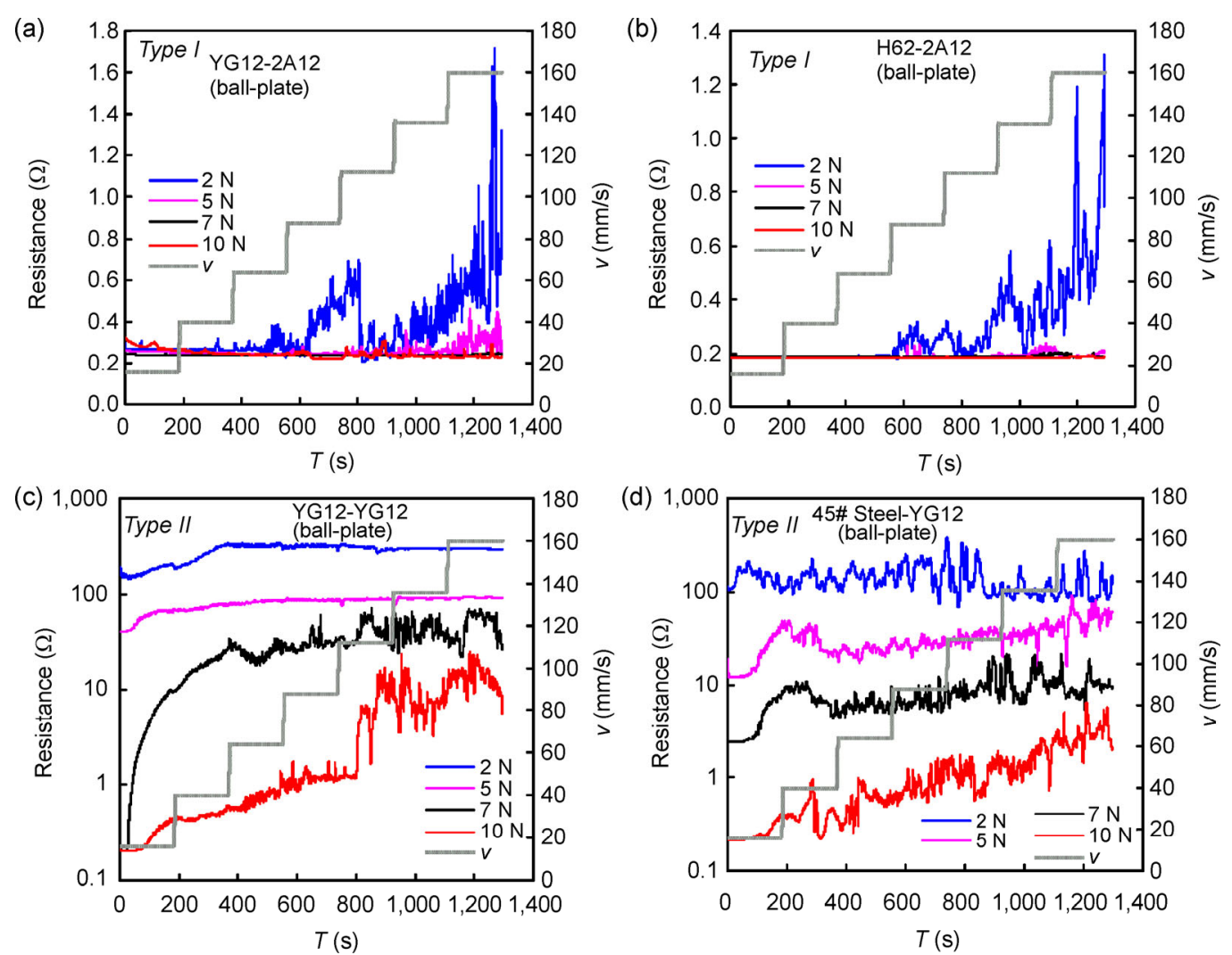

Fig. 6 Contact resistance under different loads with the increase in velocities. (a)-(d): YG12-2A12, H62-2A12, YG12-YG12, 45\# steel-YG12. 

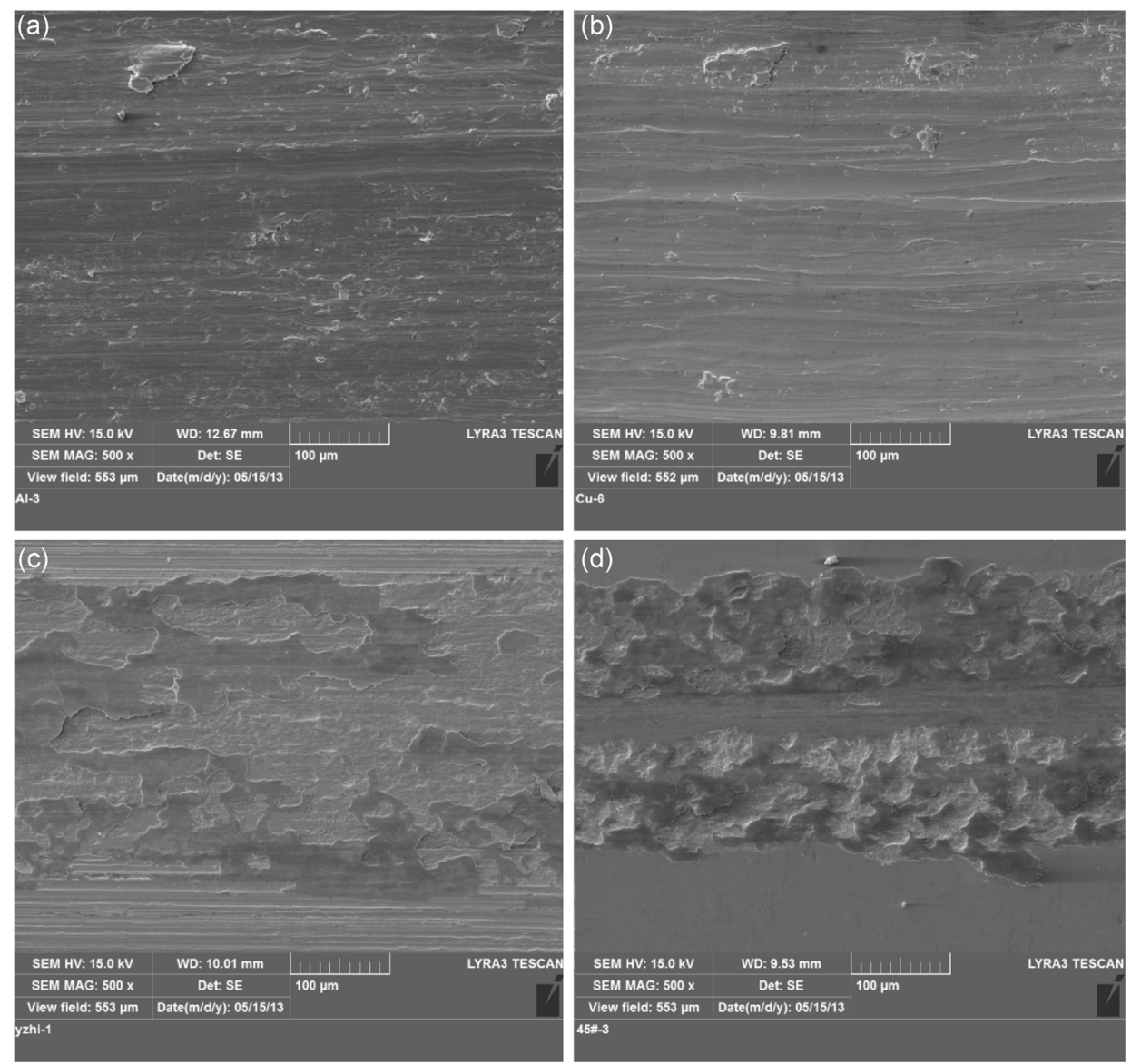

Fig. 7 SEM images of the worn surfaces of the plates. Fn=10N, 500×. (a) 2A12; (b) H62; (c) YG12; (d) $45 \#$ steel.

different wear states. As shown in Fig. 8(a), the plastic flow of the metal can be clearly seen on the wear particle surfaces of the H62-2A12 pair. These large volume grains were produced from the peeling of the adhesive points that were caused by the softening of the metal and the resulting metal flow, whereas for the Type II pair YG12-YG12 shown in Fig. 8(b), the wear particles were much smaller. This illustrates that the grains were mainly produced by the mechanical collisions and fractures of the asperities without an apparent softening or plastic flow of the metals.
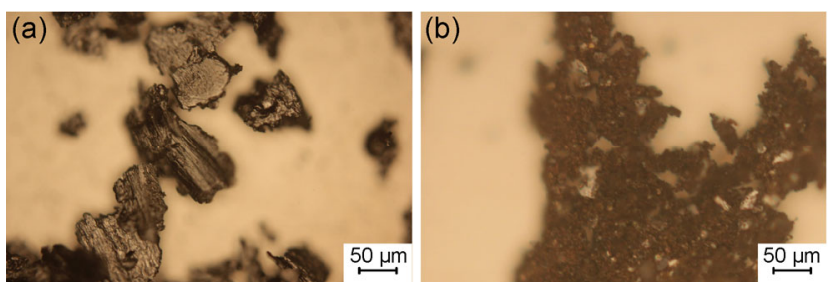

Fig. 8 Optical microscopy images of the wear particles. (a) H62$2 \mathrm{~A} 12 \mathrm{Fn}=10 \mathrm{~N}, 20 \times$; (b) YG12-YG12 Fn $=10 \mathrm{~N}, 20 \times$.
Considering the contact resistance results and the morphologies of the worn surfaces and grains, the tribological states and $\mathrm{AE}$ mechanisms for the two pair types are analyzed as follows. First, the different types may to some extent depend on the hardness of the metal pairs. At least one metal with a much lower hardness was involved for the Type I frictional pairs than that of the Type II pairs. For softer metals, plastic deformations of the asperities are more likely to occur even under a relatively low load. The AE RMS signal curves were fit with a power function, some of which are shown in Fig. 5. For the Type I pairs, the exponents were larger than 1 for all the normal loads, whereas, for the Type II pairs, the exponents were all less than 1 , which is consistent with a theoretical model based on the elastic deformation of the asperities wherein, in this model, the AE RMS is proportional to both the sliding velocity and the normal load with a power of 0.5 [27]. This consistency illustrates that the $\mathrm{AE}$ waves for the Type II pairs were more likely to 
be produced from the elastic deformations of the asperities, whereas the Type I pairs were not. On the other hand, with higher hardness and elastic modulus, the metal surfaces of the Type II pairs would undergo smaller deformations and have a smaller actual contact area under the same load than Type I, resulting in much larger contact resistances. In addition, the significant change of the contact resistance with load indicates that the asperity deformations for the Type II metal pairs may still reside in the elastic stage. With increasing sliding distance and velocity, the contact resistance slightly increased for the Type II metal pairs, particularly under high normal load, as shown in Figs. 6(c) and $6(\mathrm{~d})$. On the one hand, high sliding velocities produced an increasing number of abrasive grains, which existed between the contacting surfaces and increased the contact resistance. However, the energy dispersive X-ray spectroscopy(EDX) analysis results of the surface elements for the Type II metal pairs shown in Fig. 9 indicate that the oxygen content increased significantly after sliding, indicating that the surfaces had been oxidized during the frictional process. Clearly, the developing oxide content increased the surface electrical resistance. Accordingly, the sources of AE for the Type II metal pairs were mainly collision, elasticplastic deformation, and compression of the asperities. These processes generate both frictional force and acoustic emission waves, resulting in a positive correlation. A higher sliding speed simply resulted in more of these effects. At relatively low speeds, the collisions and fractures of the asperities dominated. These effects increased both the AE waves and the contact resistance,

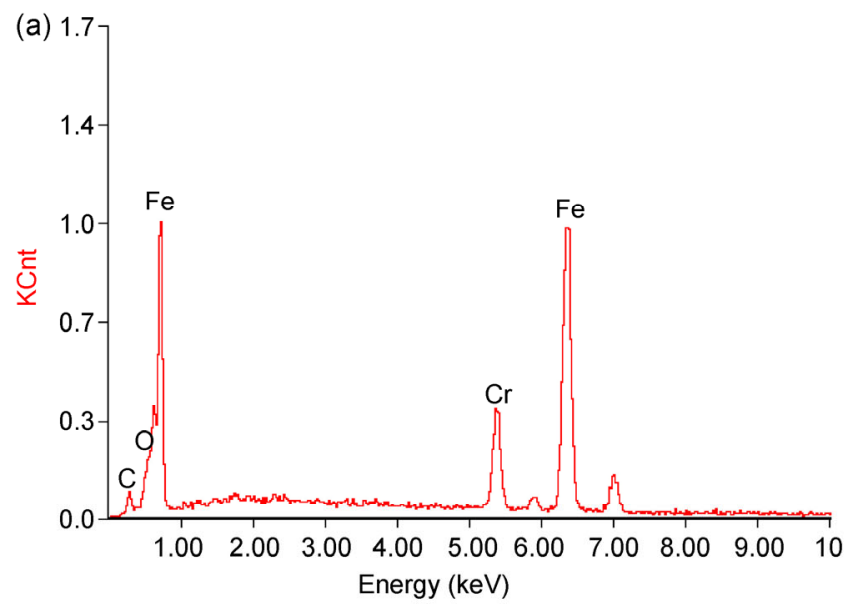

resulting in a positive $r_{\mathrm{AE}-\mathrm{RES}}$. As the sliding velocity increased, the abrasive grains played a more significant role. The separation effect of the abrasives increased the contact resistance while generating only a slightly increased AE RMS signal, whereas the compression of the grains generated $\mathrm{AE}$ waves but decreased the contact resistance. These opposite effects for the AE RMS and the contact resistance by the different processes caused the two signals to become negatively correlated. Furthermore, because of the complexity of the multiple processes involved and the large fluctuations of the contact resistance, the absolute values of $r_{\mathrm{AE}-\mathrm{RES}}$ were smaller than those of $r_{\mathrm{AE}-\mathrm{COF}}$.

In addition, the different frictional pair types may also be related to the melting point of the metal. The asperities of a metal with a low melting point more easily soften or even melt owing to high flash temperatures. For the Type I frictional pairs containing metals with lower hardness and melting points, three possible stages are proposed. At the first stage with low sliding velocity, the AE RMS and frictional coefficient were more negatively correlated whereas the contact resistance and AE RMS were more positively correlated with increasing speed. Abrasive wear dominated at this stage, leading to more impacts and compressions of the grains and, thus, greater AE signals. Moreover, the abrasives separated the two surfaces and increased the contact resistance. On the other hand, the rolling grains acting as lubrication reduced the frictional coefficient. The second stage is defined to begin at around the lowest point of $r_{\mathrm{AE}-\mathrm{COF}}$ at which the surface metal state began to change.

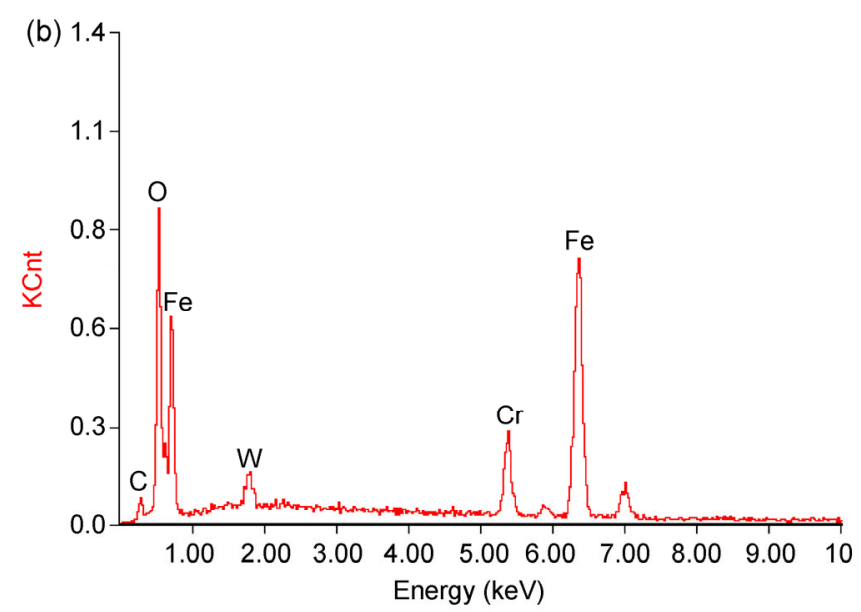

Fig. 9 EDX spectrum of the surface of YG12 plate in the Type II pair YG12-YG12. (a) before sliding; (b) after sliding. 
More heat was generated at the interface, and the asperities were softened or even melted by the high flash temperature. The increased viscosity resistance of the softened material increased the frictional force, whereas a reduced mechanical impact and better contact between the interfaces decreased the $\mathrm{AE}$ signals and the contact resistances. In the third high speed stage, $r_{\mathrm{AE}-\mathrm{COF}}$ begins to increase while $r_{\mathrm{AE}-\mathrm{RES}}$ begins to decrease. Higher temperature might further increase the mobility of the metal, and thereby slightly decrease the frictional coefficient and the AE RMS. In contrast with the Type II pairs, EDX analysis showed no obvious surface oxidation for the Type I metals. As such, the resulting decreased correlation between the contact resistance and the AE RMS might depend on the increasing resistivity caused by the high flash temperature.

Based on the above descriptions, the characteristics of $\mathrm{AE}$ and the contact resistance for the two metal frictional pair types are summarized in Table 2. Of course, these phenomena may also be related to other material properties such as the metallurgical structures, elastic modulus, etc. Here we have mainly discussed the results of our correlation analysis method and provided some preliminary analyses based on that, leaving more in-depth study regarding exact mechanisms for future work.

\section{Conclusions}

A method of multi-physical signal correlation analysis was proposed to analyze tribological states and

Table 2 Characteristics of AE and contact resistance for the two types of metal friction pairs.

\begin{tabular}{ccc}
\hline $\begin{array}{c}\text { Type of the friction } \\
\text { pair }\end{array}$ & Type I & Type II \\
\hline $\begin{array}{c}\text { Correlation of AE with } \\
\text { the friction coefficient }\end{array}$ & $\begin{array}{c}\text { For a certain range } \\
\text { of speeds: } r<1\end{array}$ & $\begin{array}{c}\text { Positive correlation } \\
r>1\end{array}$ \\
$\begin{array}{c}\text { Sensitivity of AE to } \\
\text { the normal load }\end{array}$ & Non-sensitive & Sensitive \\
$\begin{array}{c}\text { Sensitivity of contact } \\
\text { resistance to the } \\
\text { normal load }\end{array}$ & Non-sensitive & Sensitive \\
$\begin{array}{c}\text { State of the worn } \\
\text { surfaces }\end{array}$ & Plastic flow & $\begin{array}{c}\text { Elastic and plastic } \\
\text { compression and } \\
\text { partial peeling } \\
\text { YG12-YG12, } \\
\text { Typical example }\end{array}$ \\
& YG12-2A12, & H62-2A12 \\
\hline
\end{tabular}

mechanisms. Correlation curves were obtained by calculating the correlation coefficients of the multiphysical signals under varying conditions. The wear states can be quantitatively evaluated according to the different shapes of these correlation curves and the values of the correlation coefficients.

Three physical signals: acoustic emission, contact resistance, and the frictional coefficient, were selected for correlation analysis during dry sliding of four metals, which were 2A12 aluminum alloy, H62 brass, 45\# carbon steel, and YG12 carbide. The correlation coefficient curves under different loads and sliding speeds were found to be distinguishable according to two types. The correlation coefficient $r_{\mathrm{AE}-\mathrm{COF}}$ for the Type I metal pairs were negative over a wide speed range and varied non-monotonically with increasing speed. For these metal pairs, the AE RMS and the contact resistance both changed significantly with increasing normal load. In contrast, $r_{\mathrm{AE}-\mathrm{COF}}$ of the Type II metal pairs were always positive and increased monotonically with increasing speed. For the Type II metal pairs, an increasing normal load had little influence on either the AE RMS signal or the contact resistance. The correlation analysis results between several physical signals were demonstrated to correspond to different frictional behaviors and states. The anomalous correlations between the AE RMS and the frictional coefficient of different metals in various operating conditions shown in this paper should be properly considered in applications of industrial condition monitoring. In addition, the correlation analysis method undertaken here would also provide a connection point for other physical phenomena in addition to the $\mathrm{AE}$ and the contact resistance during frictional interaction and would promote the multidisciplinary development of tribological research.

\section{Acknowledgements}

This work is supported by the National Basic Research Program of China with Grant 2011CB707603 and the National Natural Science Foundation of China (Grant Nos. 51175281 and 51323006).

Open Access: This article is distributed under the terms of the Creative Commons Attribution License which 
permits any use, distribution, and reproduction in any medium, provided the original author(s) and source are credited.

\section{References}

[1] Minewitsch A A. Some developments in triboanalysis of coated machine components. Tribology in Industry 33(4): 153-158(2011)

[2] Binnig G, Quate C F, Gerber C. Atomic force microscope. Phys Rev Lett 56(9): 930 (1986)

[3] Landman U, Luedtke W D, Burnham N, Colton R J. Atomistic mechanisms and dynamics of adhesion, nanoindentation, and fracture. Science 248(4954): 454-461 (1990)

[4] Burnham N A, Dominguez D D, Mowery R L, Colton R J. Probing the surface forces of monolayer films with an atomic-force microscope. Phys Rev Lett 64(16): 1931 (1990)

[5] Hsu S, Ying C, Zhao F. The nature of friction: A critical assessment. Friction 2(1): 1-26 (2013)

[6] Mate C M, McClelland G M, Erlandsson R, Chiang S. Atomic-scale friction of a tungsten tip on a graphite surface. Phys Rev Lett 59(1942): 1942-1945 (1987)

[7] Meyer G, Amer N M. Simultaneous measurement of lateral and normal forces with an optical-beam-deflection atomic force microscope. Appl Phys Lett 57(20): 2089-2091 (1990)

[8] Krim J, Widom A. Damping of a crystal oscillator by an adsorbed monolayer and its relation to interfacial viscosity. Phys Rev B 38(17): 12184 (1988)

[9] Dayo A, Alnasrallah W, Krim J. Superconductivity-dependent sliding friction. Phys Rev Lett 80(8): 1690 (1998)

[10] Krim J, Solina D H, Chiarello R. Nanotribology of a Kr monolayer: A quartz-crystal microbalance study of atomicscale friction. Phys Rev Lett 66(2): 181-184 (1991)

[11] Smith K C A, Oatley C W. The scanning electron microscope and its fields of application. Brit J Appl Phys 6(11): 391(1955)

[12] Schmit J, Creath K, Wyant J C. Surface profilers, multiple wavelength, and white light interferometry. Optical Shop Testing: 667-755 (2007)

[13] Renouf M, Massi F, Fillot N, Saulot A. Numerical tribology of a dry contact. Tribol Int 44(7): 834-844 (2011)

[14] Blok H. The flash temperature concept. Wear 6(6): 483-494 (1963)

[15] Archard J F. The temperature of rubbing surfaces. Wear 2(6): 438-455 (1959)
[16] Ibrahim R A. Friction-induced vibration, chatter, squeal, and chaos-part II: dynamics and modeling. Appl Mech Rev 47(7): 227-253 (1994)

[17] Harper W R. Contact and Frictional Electrification. Laplacian Press, 1967.

[18] Walton A J. Triboluminescence. Adv Phys 26(6): 887-948 (1977)

[19] Shizhu W, Ping H. Principles of tribology. Beijing (China): Tsinghua University Press, 2008.

[20] Scruby C B. An introduction to acoustic emission. J Phys E: Sci Instrum 20(8): 946 (1987)

[21] Ravindra H V, Srinivasa Y G, Krishnamurthy R. Acoustic emission for tool condition monitoring in metal cutting. Wear 212(1): 78-84 (1997)

[22] Douglas R M, Steel J A, Reuben R L. A study of the tribological behaviour of piston ring/cylinder liner interaction in diesel engines using acoustic emission. Tribol Int 39(12): 1634-1642 (2006)

[23] Hase A, Wada M, Mishina H. Correlation of material transfer phenomena and AE signals in adhesive wear. Jpn J Tribol 50(6): 717-726 (2005)

[24] Hase A, Wada M, Mishina H. Correlation of abrasive wear phenomenon and AE signals. Jpn J Tribol 51(5): 639-651 (2006)

[25] Hase A, Wada M, Mishina H. Acoustic emission signals and wear phenomena on severe-mild wear transition. Tribol Onl 3(5): 298-303 (2008)

[26] Hase A, Mishina H, Wada M. Correlation between features of acoustic emission signals and mechanical wear mechanisms. Wear 292: 144-150 (2012)

[27] Fan Y, Gu F, Ball A. Modelling acoustic emissions generated by sliding friction. Wear 268(5): 811-815 (2010)

[28] Baranov V M, Kudryavtsev E M, Sarychev G A. Modelling of the parameters of acoustic emission under sliding friction of solids. Wear 202(2): 125-133 (1997)

[29] Archard J F. Contact and rubbing of flat surfaces. J Appl Phys 24(8): 981-988 (2004)

[30] Holm R, Holm E. Electric Contacts: Theory and Application. New York (UK): Springer-Verlag, 1967.

[31] Kogut L, Komvopoulos K. Electrical contact resistance theory for conductive rough surfaces. J Appl Phys 94(5): 31533162 (2003)

[32] Greenwood J A. Constriction resistance and the real area of contact. Brit J Appl Phys 17(12): 1621 (1966) 


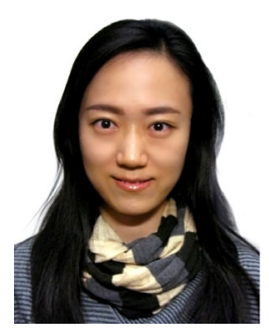

Pengyi TIAN. She received her Bachelor degree in Mechanical Engineering in 2011 from Shandong University, Weihai, China. Now

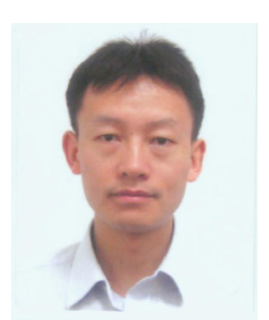

Yu TIAN. He is Professor and Vice Director of the State Key Laboratory of Tribology at Tsinghua University of China. Tian gained his BA and PhD degrees in Mechanical Engineering at Tsinghua University in 1998 and 2002, respectively. Subsequently he joined the State Key Laboratory of Tribology. He did his postdoc at University of California, Santa Barbara with Professor Jacob Israelachvili (from 2005 to 2007), and a visiting associate professor at Nanyang Technology University for five months. His research she is a PhD candidate in the State Key Laboratory of Tribology at Tsinghua University. Her research is focused on the multi-physics during friction and stick-slip process. interest is the science and technology at the interface of physics, materials, engineering and biology to understand the physical laws of adhesion, friction and rheology. He has published over 90 peer-reviewed journal papers. He has received the Wen ShizhuMaple Award-Young Scholar Award (2012), the Young Scholar Achievement Award of the Society of Mechanical Engineering of China (2011), Outstanding Young Scholar Award of the Chinese Tribology Institute (2009), and the National Excellent Doctoral Dissertation of China (2004). 